Research in Astronomy and Astrophysics manuscript no.

(LTEX: RAA-2018-0185.R1.tex; printed on December 12, 2018; 2:47)

\title{
Strange quark star in dilaton gravity
}

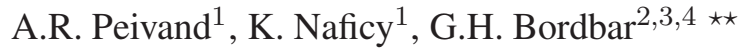 \\ ${ }^{1}$ Department of Physics, University of Birjand, PO Box 615/97175, Birjand, South Khorasan, Iran; \\ naficy@birjand.ac.ir \\ 2 Department of Physics, Shiraz University, Shiraz 71454, Iran $^{\dagger}$ \\ ${ }^{3}$ Research Institute for Astronomy and Astrophysics of Maragha, P.O. Box 55134-441, Maragha, Iran \\ ${ }^{4}$ Department of Physics and Astronomy, University of Waterloo, 200 University Avenue West, Waterloo, \\ Ontario, N2L3G1, Canada
}

Received 20XX Month Day; accepted 20XX Month Day

\begin{abstract}
In this work, we have first obtained the hydrostatic equilibrium equation in dilaton gravity. Then we have examined some of the structural characteristics of strange quark star in dilaton gravity with a background of Einstein gravity. We have shown that the variations of dilaton parameter do not affect the maximum mass of quark star, while the variations of the cosmological constant lead to change in the structural characteristics of the quark star. We have investigated the stability of strange quark stars that studied by MIT Bag model, in dilaton gravity. We have also provided limiting values for the dilaton field parameter and the cosmological constant.We have also studied the effects of dilaton gravity on the other properties of quark star such as the mean density and gravitational redshift. We have concluded that the last reported value for cosmological constant does not affect on maximum mass of strange quark star.
\end{abstract}

Key words: strange quark star — dilaton gravity — structure — Bag model

\section{INTRODUCTION}

Einstein's general relativity (GR) theory well explains phenomena and events within the solar system. Tolman, Oppenheimer and Volkoff (TOV) (Tolman 1939; Oppenheimer \& Volkoff 1939) obtained the first hydrostatic equilibrium equation(HEE) from the solution of the Einstein field equation. So far, the structure of compact objects, such as neutron stars and quark stars, that have general relativistic properties due to their great density has been obtained by many authors (Silbar \& Reddy 2004; Bordbar et al. 2006; Bordbar et al. 2016; Narain et al. 2006) through the numerical solution of the TOV equation.

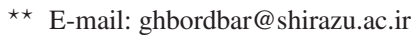


In recent years, studies outside the solar system have led to the emergence of new theories and observations such as accelerated expansion of the universe (Knop et al. 2003; Perlmutter et al. 1999; Riess et al. 1998; Tonry et al. 2003), which GR is incapable of explain, and also the absence of a comprehensive gravitational theory in quantum scales has led to a lot of attention to the emergence of new gravitational theories. Theories that include GR and can explain the new cosmological phenomena and discoveries that GR cannot explain and interpret well.

Adding new terms, including higher-order curvature invariants and scalar fields, to Einstein Lagrangian yields theories known as modified gravity. One of these new modified gravities is to consider the dilaton scalar field and its potential in the universe. The scalar field was used to justify the inflation phenomenon and also to describe cold dark matter (Cho 1990), which is a type of dark matter. Recently, the study of the structure of compact objects in modified gravity such as neutron stars (Astashenok 2016; Hendi et al. 2015), black holes (Chan et al. 1995; Hendi et al. 2016) and quark stars (Astashenok 2016; Astashenok et al. 2015) has been widely considered.

In order to obtain the structural characteristics of compact objects in the new gravities, we use the action related to the proposed gravitational theory. By varying this action and taking the principle of least action into account, we obtain the field equation for the considered gravity. By solving this equation and using the conservation of the momentum-energy tensor, we obtain HEE of the new theory. Then, by using the equation of state $(\mathrm{EoS})$ of the matter of the compact object, we solve the obtained HEE equation numerically and obtain the maximum mass and radius of the considered star. The HEE in modified gravity is considered by several authors: HEE in Gauss-Bonnet gravity (Momeni \& Myrzakulov 2015), HEE in $f(G)$ gravity (Sharif \& Fatima 2016), HEE in $f(R)$ gravity (Astashenok 2016; Astashenok et al. 2013; Arapoğlu et al. 2011), HEE in gravity's rainbow (S.H. et al. 2016), HEE in massive gravity (Katsuragawa et al. 2016; Hendi et al. 2017)

After Witten conjectured hypothesis of strange quark matter (Witten 1984; Farhi \& Jaffe 1984), it has been shown that a new class of compact object may exist that have been composed from strange quark matter, known as strange quark stars(SQS) or strange stars(SS) (Haensel et al. 1986; Alcock et al. 1986b,a; Alcock \& Olinto 1988; Glendenning 1990). Strange quark stars composed from up $(u)$, down $(d)$ and strange $(s)$ quarks along with a small number of electrons that are in $\beta$-equilibrium. The best candidates for being SQS are some of the observed compact objects that could not be compatible with neutron star model, such as X-ray pulsar LMC X-4 with $M=1.29 \pm 0.05 M_{\odot}$ (Gangopadhyay et al. 2013; Paul et al. 2011; Rawls et al. 2011), X-ray burster 4U 1608-52 (Paul et al. 2011; Bombaci 1997; Dey et al. 1998) with $M=1.74 \pm 0.14 M_{\odot}$ (Güver et al. 2010) and millisecond pulsar J1614-2230 (Paul et al. 2011; Deb et al. 2017) with $M=1.97 \pm 0.04 M_{\odot}$ (Demorest et al. 2010). In this work, the considered quark star is made of strange quark matter from the center up close to the surface. Here, we want to calculate some bulk properties of SQS by using modified hydrostatic equilibrium equation in dilaton gravity and considering MIT Bag model to obtain equation of state of SQM. This paper is divided into six sections. In Sec. 2, we propose a new procedure to find HEE in dilaton gravity on the background of Einstein gravity. In Sec. 3, we present the HEE in dilaton gravity as a correction of the HEE in Einstein gravity(correction of TOV). 
in Secs. 2 and 3 and compare our results with the results obtained in GR. In Sec. 5, we have investigate causality condition and dynamical stability of SQS in dilaton gravity. Our conclusions are drawn in the 6th section.

\section{HEE IN DILATON GRAVITY}

The total action of dilaton gravity $\left(S_{\text {total }}\right)$ is as follows

$$
S_{\text {total }}=\frac{1}{2 \kappa} \int d^{4} x \sqrt{-g}\left(R-2 g^{\mu \nu} \partial_{\mu} \Phi \partial_{\nu} \Phi-V(\Phi)\right)+S_{\text {matter }},
$$

in which Einstein's gravity is given as background. In this action, $R, \Phi$ and $V(\Phi)$ are Ricci scalar, dilaton field and the potential of this field, respectively. $S_{\text {matter }}$ is the action related to the matter, which is considered to be a perfect fluid. In order to find the dilaton field equation, we vary Eq. 1 with respect to the dilaton field $\Phi$ and the metric tensor $g_{\mu \nu}$. Using the least action principle, field equations are obtained as follows

$$
\begin{gathered}
G_{\mu \nu}=\kappa T_{\mu \nu}+\left(2 \partial_{\mu} \Phi \partial_{\nu} \Phi-g_{\mu \nu} \partial_{a} \Phi \partial^{a} \Phi-\frac{1}{2} g_{\mu \nu} V(\Phi)\right) \\
\square \Phi-\frac{1}{4} \frac{\partial V}{\partial \Phi}=0
\end{gathered}
$$

Where $G_{\mu \nu}$ and $\kappa=\frac{8 \pi G}{c^{4}}$ are the Einstein tensor and Einstein gravitational constant, respectively. $T_{\mu \nu}$ is the energy-momentum tensor associated with the perfect fluid. We consider that the potential of dilaton field consists of two Liouville terms.

$$
V(\Phi)=2 \Lambda_{0} e^{2 \xi_{0} \Phi}-2 \Lambda e^{2 \xi \Phi}
$$

Potentials of this type have been previously studied to solve dilaton black hole field equations (Chan et al. 1995; Dehghani \& Farhangkhah 2005).

In order to find a static solution of Eqs. 2 and 3, we assume the four-dimensional spacetime metric as

$$
d s^{2}=-B(r) d t^{2}+\frac{d r^{2}}{A(r)}+R^{2}(r) r^{2} d \Omega^{2}
$$

in which $B(r), A(r)$, and $R(r)$ are unknown functions to be determined and $d \Omega^{2}=\left(d \theta^{2}+\sin ^{2} \theta d \phi^{2}\right)$. We consider $R(r)$ as the ansatz which has the form

$$
R(r)=e^{\alpha \Phi(r)}
$$

This ansatz was first used to investigate answers of field equations of charged dilaton black strings (Dehghani \& Farhangkhah 2005). Recently, this ansatz has been used by some authors (Sheykhi et al. 2006; Hendi et al. 2015) to study properties of dilaton gravity. It is important to note that when $\alpha=0$ the ansatz becomes $R(r)=1$ and dilaton gravity turns to Einstein gravity. By making this ansatz and using Eq. 3 and introduced metric in Eq. 5 we have

$$
\Phi(r)=\frac{\alpha}{1+\alpha^{2}} \ln \left(\frac{b}{r}\right)
$$

where $\mathrm{b}$ is an arbitrary constant.

For a perfect fluid, the general form of energy-momentum tensor is given by 
In these relations, $P$ and $\rho$ are the pressure and energy density of the perfect fluid from the viewpoint of the local observer respectively and $u^{\mu}$ is the local fluid velocity 4-vector. Using the metric Eqs. 5and 8, the energy-momentum tensor for the perfect fluid has the form

$$
T_{b}^{a}=\operatorname{diag}\left(-\rho c^{2}, P, P, P\right) .
$$

To solve dilaton field equation, with the metric Eq. 5 and energy-momentum tensor Eq. 9, we obtain the components of Eq. 2 as follows

$$
\begin{aligned}
\kappa \rho c^{2} & =-\Lambda \gamma^{\alpha^{2}}-\frac{A^{\prime}}{r K_{1,1}}-\frac{A K_{1,-3}}{r^{2} K_{1,1}^{2}}+\frac{\gamma \alpha^{2}}{K_{-1,1} b^{2}}+\frac{1}{r^{2} \gamma^{\alpha^{2}}} \\
\kappa P & =\Lambda \gamma^{\alpha^{2}}+\frac{A B^{\prime}}{r B K_{1,1}}+\frac{A K_{1,-3}}{r^{2} K_{1,1}^{2}}-\frac{\gamma \alpha^{2}}{K_{-1,1} b^{2}}-\frac{1}{r^{2} \gamma^{\alpha^{2}}} \\
\kappa P & =\Lambda \gamma^{\alpha^{2}}+\frac{A B^{\prime}}{2 r B K_{1,1}}-\frac{2 \alpha^{2} A}{r^{2} K_{1,1}^{2}}-\frac{\gamma \alpha^{2}}{K_{-1,1} b^{2}}-\frac{A B^{\prime 2}}{4 B^{2}} \\
& +\frac{A^{\prime} B^{\prime}}{4 B}+\frac{A^{\prime}}{2 r K_{1,1}}+\frac{A B^{\prime \prime}}{2 B}
\end{aligned}
$$

where

$$
K_{i, j} \equiv i+j \alpha^{2}, \quad \gamma \equiv\left(\frac{b}{r}\right)^{\frac{2}{K_{1,1}}}
$$

and the prime and double-prime are the keys that denote the first and second derivatives with respect to $r$ respectively. Furthermore, by using the conservation of the energy-momentum tensor $T_{; \mu}^{\mu \nu}=0$ we have

$$
\frac{d P}{d r}=-\left(\rho c^{2}+P\right)\left(\frac{B^{\prime}}{B}\right)
$$

It is notable that in the equations 10 to 12 , we set $\Lambda$ to be a free parameter and behaves same as cosmological constant. On the other hand, $\Lambda_{0}, \xi_{0}$ and $\xi$ are constants that are chosen as follows to solve Eqs. 2 and 3 ,

$$
\Lambda_{0}=\frac{\alpha^{2}}{b^{2} K_{-1,1}}, \quad \xi_{0}=\frac{1}{\alpha}, \quad \xi=\alpha .
$$

The function $A(r)$ is obtained by integrating Eq. 10 as the form

$$
\begin{aligned}
A(r)= & \left(\frac{\gamma^{-\alpha^{2}}}{r^{2} K_{1,-1}}-\frac{\Lambda \gamma^{\alpha^{2}}}{3 K_{1,-1}}-\frac{\gamma \alpha^{2}}{K_{1,-1}^{2} b^{2}}\right) r^{2} K_{1,1}^{2} \\
& -\frac{\kappa c^{2} K_{1,1}}{r^{\frac{K_{1,-3}}{K_{1,1}}}} \int r^{\frac{2 K_{1,-1}}{K_{1,1}}} \rho(r) \mathrm{d} r
\end{aligned}
$$

If we replace $\alpha=0$ in this equation we see that $A(r)$ in dilaton field reduces to known $A(r)=\left(1-\frac{2 G M}{r c^{2}}-\right.$ $\left.\frac{\Lambda}{3} r^{2}\right)$ in Einstein- $\Lambda$ gravity (Stuchlík 2000; Balaguera-Antolínez et al. 2005; Böhmer \& Harko 2005a), therefore, we can write $4 \pi \int r^{\frac{2 K_{1,-1}}{K_{1,1}}} \rho(r) \mathrm{d} r=M_{e f f}(r, \alpha)$ that $M_{e f f}(r, \alpha)=\int 4 \pi \rho(r) R_{e f f}^{2} \mathrm{~d} R_{e f f}$ is the effective mass with the corresponding effective radius that given by $R_{\text {eff }}=\sqrt[3]{\frac{3 K_{1,1}}{K_{3,-1}}} r\left(\frac{K_{3,-1}}{3 K_{1,1}}\right)$. One can obtain $\frac{B^{\prime}}{B}$ by substituting Eq. 14 into Eq. 11 , then replace $\frac{B^{\prime}}{B}$ into Eq. 13. With this replacement and using $\frac{d P}{d r}=\frac{d P}{d R_{e f f}} \frac{d R_{e f f}}{d r}$ we obtain dilaton HEE as

$$
\begin{aligned}
\frac{d P}{d R_{e f f}} & =\frac{1}{2} \sigma^{\frac{4 \alpha^{2}}{3 K_{1,1}}} \delta^{-\frac{2 K_{1,-1}}{K_{1,1}}}\left(c^{2} \rho+P(r)\right)\left[-\frac{\sigma \delta K_{1,1} P(r) \kappa}{\tau}\right. \\
& \left.-\frac{K_{1,1}}{\tau}+\frac{\sigma \delta K_{1,1} \Lambda Y^{\alpha^{2}}}{+}+\frac{\sigma \delta K_{1,1} \alpha^{2} Y}{}+\frac{K_{1,-3}}{}\right]
\end{aligned}
$$




$$
\begin{gathered}
\text { with } \tau=\frac{K_{1,1}^{2}}{\Upsilon^{\alpha^{2}} K_{1,-1}}-\frac{K_{1,1}^{2} \delta^{2} \sigma^{2} \Upsilon \alpha^{2}}{K_{1,-1}^{2} b^{2}}-\frac{K_{1,1}^{2} \delta^{2} \sigma^{2} \Lambda \Upsilon^{\alpha^{2}}}{3 K_{1,-1}}-\frac{\kappa c^{2} K_{1,1} M_{\text {eff }}}{4 \pi\left((\sigma \delta)^{\frac{K_{1,-3}}{K_{1,1}}}\right)}, \\
\Upsilon=\left(\frac{b}{\sigma \delta}\right)^{\frac{2}{K_{1,1}}}, \quad \delta=R_{\text {eff }}^{\left(\frac{3 K_{1,1}}{K_{3,-1}}\right)}, \quad \sigma=\left(\frac{K_{3,-1}}{3 K_{1,1}}\right)^{\left(\frac{K_{1,1}}{K_{3,-1}}\right)} .
\end{gathered}
$$

It is important to note that in dilaton gravity, gravitational mass, $m(r)=\int 4 \pi r^{2} \rho(r) d r$, and radius, $r$, are modified to $M_{e f f}$ and $R_{e f f}$ respectively.

\section{DILATON HEE AS A CORRECTION OF TOV}

In this section, we use the same techniques as in the Ref. Hendi et al. 2015. We expand Eq. 15 in series of $\alpha$. When $\alpha$ has very small values, we can neglect all terms of order higher than 2 , then we have

$$
\begin{aligned}
\frac{d P}{d r}= & \frac{\left(4 r^{3} P(r) \kappa \pi-\frac{8}{3} \Lambda \pi r^{3}+\kappa c^{2} m(r)\right)\left(\rho c^{2}+P(r)\right)}{2 r\left(\kappa c^{2} m(r)+\frac{4}{3} r \pi\left(\Lambda r^{2}-3\right)\right)} \\
& -\alpha^{2} \frac{72\left(\rho c^{2}+P(r)\right)}{r\left(4 \Lambda \pi r^{3}+3 \kappa c^{2} m(r)-12 r \pi\right)^{2}}\left\{\frac{\pi r}{3} \ln \left(\frac{b^{2}}{r^{2}}\right)\right. \\
& \times\left[\frac{3}{4} \kappa c^{2}\left(\Lambda r^{2}+1\right) m(r)+\pi\left[\kappa\left(\Lambda r^{2}+3\right) P(r)-4 \Lambda\right] r^{3}\right] \\
& +\frac{1}{4} \kappa^{2}(m(r))^{2} c^{4}+\pi c^{2} \kappa m(r) r\left[\kappa P(r) \ln (r) r^{2}+\left(-\Lambda r^{2}\right.\right. \\
& \left.+1) \ln (r)+\frac{2}{3} \Lambda r^{2}-\frac{7}{4}\right]+\pi^{2} r\left[r ^ { 2 } \kappa P ( r ) \left(\kappa c^{2} N(r)+\frac{2}{3} \Lambda r^{3}\right.\right. \\
& \left.\left.-r)-\kappa c^{2} N(r)\left(\Lambda r^{2}-1\right)-\frac{2}{9} r^{5} \Lambda^{2}-\frac{2}{3} \Lambda r^{3}+2 r\right]\right\}
\end{aligned}
$$

in which $N(r)=\int-4 r^{2} \ln (r) \rho(r) \mathrm{d} r$. As it can be seen, the first term on the right-hand side of this equation is the well-known TOV equation, and the second term is considered as a correction term in dilaton gravity. A close inspection of Eq. 16, in the case of $\alpha=0$, the new HEE equation will be exactly the same as TOV.

\section{STRUCTURE OF STRANGE QUARK STARS IN DILATON GRAVITY}

In this section, we calculate some structural properties of strange quark stars(SQS) in dilaton gravity. In order to obtain the configurational characteristics of the quark stars, we must solve the HEE equation, Eq. 16, numerically by using an $\operatorname{EoS}$ of the form $P=P(\rho)$.

\subsection{EoS of strange quark matter}

We obtain EoS of strange quark matter (SQM) using the MIT bag model. In this model, the total energy is the sum of kinetic energy of quarks plus a bag constant ( $B_{b a g}$ ) (Chodos et al. 1974). In fact, the bag constant $B_{b a g}$ can be interpreted as the difference between the energy densities of the noninteracting quarks and the interacting ones. Dynamically it acts as a pressure, that keeps the quark gas in constant density and potential. The value of bag constant, $B_{b a g}$, lies in the interval $58.8 \frac{\mathrm{MeV}}{\mathrm{fm}^{3}}<B_{b a g}<91.2 \frac{\mathrm{MeV}}{\mathrm{fm}^{3}}$ (Stergioulas 2003) that in this work, the values 60,75 and $90 \frac{\mathrm{MeV}}{\mathrm{fm}^{3}}$ are considered for the bag constant. In order to obtain the equation of state, we have neglected the mass of $u$ and $d$ quarks and considered the mass of $s$ quark to be 
Fig. 1 we have plotted behavior of EoS of SQM in MIT Bag model for various values of $B_{b a g}$. This figure shows that for all values of bag constant EoS of SQM has linear treatment. Also, it is observed that EoS for SQM with a lower value of $B_{b a g}$ is stiffer than the higher case. In the next section, we calculate maximum mass and corresponding radius of SQS in dilaton gravity by using this EoS and Eq. 16 (also Eq. 15).

\subsection{Maximum mass of SQS in dilaton gravity}

Like other compact objects, SQSs reach a limit gravitational mass known as the maximum mass. Subsequently, we can obtain the maximum mass $\left(M_{\max }\right)$ of SQS in dilaton gravity in the states with various values of $\alpha$ and $\Lambda$, by numerically integrating of Eq. 16 (or Eq. 15) and using EoS of SQM.

In Tab. 1, We have calculated and presented structural properties of SQS for variation of $\alpha$ with the reported value of the cosmological constant $\left(\Lambda \leq 10^{-56} \mathrm{~cm}^{-2}\right)$ and for vanishing cosmological constant. As we can see these values of $\Lambda$ and $\alpha$ have not the significant impact on the structural properties of SQS.

It is worth mentioning that finding the exact value of the cosmological constant is an open issue. Although, its reported value can be valid in large-scale structure, but when we encounter with a local scale structure in close to a massive object such as neutron stars or SQSs, the value of $\Lambda$ may be very different from its reported value for large-scale structure (Bordbar et al. 2016). Motivated by this reason, $\Lambda \leq 10^{-13} \mathrm{~cm}^{-2}$ has been applied to study of structural properties of compact object in the presence of cosmological constant by several authors (Hendi et al. 2015; Bordbar et al. 2016; Zubairi et al. 2015). Also, it is notable that some of the cosmological models, consider $\Lambda$ as a decreasing variable with time, that has been predicted by decaying vacuum energy (Waga 1993; Böhmer \& Harko 2005b). Therefore, considering the effect of such different values of the cosmological constant on structural properties of a compact object can be interesting. In the remainder of this section, we have used $\Lambda \leq 10^{-13} \mathrm{~cm}^{-2}$ as well, to obtain bulk properties of SQSs in dilaton gravity.

In Fig. 2, we have plotted the gravitational mass-radius for $\Lambda=\alpha=0$. The values of the maximum mass and the corresponding radius values are listed in Tab. 2. As we can see, these values are the same as $M_{\max }$ and R in Einstein gravity (Bordbar \& Peivand 2011).

The gravitational mass versus radius and central energy density for a constant value of $\alpha$ but in different values of $\Lambda$ is plotted in Figs. 3 and 5, respectively. We have also plotted the effective gravitational mass versus effective radius (see Fig. 4). Fig. 3 shows that increasing in $\Lambda$ has led to an increase in the maximum mass. Moreover, for $\Lambda \leq 10^{-14}$ the deviation of $M-R$ relation from Einstein gravity is very small (see Fig. 3). This result is repeated in Fig. 4 and can be seen values of the gravitational mass and corresponding radius are same as effective mass and corresponding effective radius (see Tab. 2). For a given central energy density $\left(\rho_{c}\right)$, the mass of SQS increases by increasing cosmological constant (see Fig. 5). We can see that for higher values of the cosmological constant, increasing gravitational mass has a higher rate and reaching to $M_{\max }$ occurs in SQSs with smaller values of central energy density $\left(\rho_{c}\right)$.

In Fig. 6, we investigate the quark star structure for a given positive value of $\Lambda$ but for different values of $\alpha$, which shows that dilaton parameter, $\alpha$, variations do not affect in SQS structure. It should be noted 
$B_{b a g}=90 \frac{\mathrm{MeV}}{\mathrm{fm}^{3}}$ is considered in figures drawn so far. In Fig. 7, we have plotted mass-radius relation of SQS in dilaton gravity for $B_{b a g}=60$ and $75 \frac{\mathrm{MeV}}{\mathrm{fm}^{3}}$ for a given value of $\Lambda$ and different values of $\alpha$. It can be seen that in these situations, the lack of influence of $\alpha$ on the mass and the radius of SQS is still remained. The values of the maximum mass and corresponding radius values in these modes are shown in Tabs. 3 and 4 .

In Fig. 8 the maximum gravitational mass as a function of $\Lambda$ in all cases of $B_{b a g}$ is plotted. It shows that $M_{\max }$ is an increasing function of $\Lambda$, however, the rate of the increase of $M_{\max }$ versus $\Lambda$ increases with increasing bag constant.

In Fig. 9, we have plotted the energy per baryon $(E / A)$ for SQM with the various values of Bag constant as a function of pressure. We see that the zero point of pressure for SQM considered with lower values of $B_{b a g}$ has a lower E/A. This implies that SQMs considered with lower values of $B_{b a g}$ are more stable than other ones. In Tab. 5, we have obtained the percentage increase of maximum mass of SQS and we have considered various values of the bag constant to calculate EoS of SQM in different values of $\Lambda$ with respect to obtained the $M_{\max }$ in GR $(\alpha=\Lambda=0)$ with same value of $B_{\text {bag }}$ (For instance $(\Delta M)_{\Lambda_{1}}^{B=90}=$ $\left(M_{\max }\right)_{\Lambda_{1}=1 \times 10^{-13}}^{B=90}-\left(M_{\max }\right)_{\Lambda_{0}=0}^{B=90}=0.02 M_{\text {sun }}=1.49 \%\left(M_{\max }\right)_{\Lambda_{0}=0}^{B=90}$ obtained for $\left.B_{\text {bag }}=90\right)$. We can see that effect of $\Lambda$ on SQSs that have higher stability is upper than other ones(see columns of Tab. 5). In addition, this table shows that increasing rate of the percentage increase of maximum mass of SQS, enhances by enhancing $\Lambda$ (see rows of Tab. 5).

Negative values of $\Lambda$ are investigated in Fig. 10 in a particular value of $\alpha$ to obtain SQS structure. As we can see, increasing the maximum mass and its corresponding radius is simultaneous with increasing in cosmological constant in negative values ( also see Tab. 6).

It should be noted that for $\Lambda \geq 10^{-12}$, HEE in dilaton gravity does not have a logical answer. The structural results for $\Lambda<10^{-14}$ are similar to those for $\Lambda=0$.

\subsection{Redshift and mean density}

The information about the compactness of a compact object, mass-radius ratio, can be found from the gravitational redshift that is an observational quantity(denoted by $z$ ). We can obtain $z$, by using Eq. 14 in two forms

$$
\begin{array}{r}
z_{e f f}=\left\{\left[-\frac{\Lambda(\Upsilon)^{\alpha^{2}}}{3 K_{1,-1}}-\frac{\Upsilon \alpha^{2}}{K_{1,-1}^{2} b^{2}}+\frac{(\Upsilon)^{-\alpha^{2}}}{\Omega^{2} K_{1,-1}}\right] \Omega^{2} K_{1,1}^{2}\right. \\
\left.-\frac{2 G K_{1,1} M_{e f f} \Omega^{-\frac{K_{1,-3}}{K_{1,1}}}}{c^{2}}\right\}^{-\frac{1}{2}}-1
\end{array}
$$

and

$$
\begin{array}{r}
z=\left\{\left[1-\frac{2 G M}{R c^{2}}-\frac{\Lambda R^{2}}{3}\right]+\frac{\alpha^{2}}{3 R c^{2}}\left[-R c^{2}\left(R^{2} \Lambda+3\right) \ln \left(\frac{b^{2}}{R^{2}}\right)\right.\right. \\
\left.\left.+\left(-3 \Lambda R^{3}+6 R\right) c^{2}-14 G M\right]\right\}^{-\frac{1}{2}}-1
\end{array}
$$


where in Eq. 17, $\Omega=\sigma \delta$ and $\Upsilon$ are functions of $R_{\text {eff }}$ and to obtain Eq. 18, we have expanded Eq. 14 in series of $\alpha$ and neglected all terms of order higher than 2 .

In Tables 2 to 4 we have evaluated $z$ and $\bar{\rho}=\frac{3 M}{4 \pi R^{3}}$ (the mean energy density) for SQS in different cases. For all cases, we can see that with increasing $\Lambda$, gravitational redshift increases while $\bar{\rho}$ decreases (see Tabs. 2 and 4). We can see that increasing in $\Lambda$ is equivalent to increasing $B_{b a g}$. Therefore, it can be interpreted as increasing the values of the difference between the energy densities of non-interacting quarks and interacting ones. In addition, we can see that for fixed values of $\Lambda$, variations of $\alpha$ do not affect on $z$ and $\bar{\rho}$.

\subsection{Energy conditions}

Any acceptable physical model of isotropic fluid must satisfy conditions of energy (S.H. et al. 2016; Visser \& Barcelo 2000). The variation of pressure and energy density of a SQS with respect to fractional radius are shown in Figs. 11 and 12. As we can see the pressure of system is non-zero and positive at origin. Also, it is clear that the pressure and energy density decrease monotonically by increasing fractional radius. Moreover, It is observed that pressure reaches to zero at the boundary of the $\operatorname{star}(r=R)$ in all cases. Also, sum of pressure and energy density versus $\frac{r}{R}$ throughout of star are seen in these figures. It is clear that all energy conditions for a perfect fluidare satisfied for SQS in dilaton gravity.

\subsection{Matching interior and exterior solutions}

In this part, we investigate interior and exterior solutions matching at the boundaries by noting that we have assumed $\rho=\bar{\rho}$ throughout of star. At first, we can find the interior radial component of metric tensor $\left(\frac{1}{A(r)}\right)$ by expanding Eq. 14 in series of $\alpha$ and neglecting all terms of order higher than 2, therefore for $r<R$ we have

$$
\begin{aligned}
A(r)_{i n}=\left[1-\frac{\Lambda r^{2}}{3}-\frac{\kappa c^{2} m(r)}{4 \pi r}\right]+ & {\left[2-\frac{1}{3} \Lambda r^{2} \ln \left(\frac{b^{2}}{r^{2}}\right)-\Lambda r^{2}-\ln \left(\frac{b^{2}}{r^{2}}\right)-\frac{\kappa c^{2} m(r)}{4 \pi r}\right.} \\
& \left.+\frac{4}{3} \kappa \bar{\rho} c^{2} r^{2} \ln (r)-\frac{4}{9} \kappa \bar{\rho} c^{2} r^{2}-\frac{\kappa c^{2} m(r) \ln (r)}{\pi r}\right] \alpha^{2}
\end{aligned}
$$

that by replacing $m(r)=\frac{M}{R^{3}} r^{3}$ and $\bar{\rho}=\frac{3 M}{4 \pi R^{3}}$ gets

$$
A(r)_{\text {in }}=\left[1-\frac{\Lambda r^{2}}{3}-\frac{\kappa c^{2} M}{4 \pi R^{3}} r^{2}\right]+\left[2-\frac{1}{3} \Lambda r^{2} \ln \left(\frac{b^{2}}{r^{2}}\right)-\Lambda r^{2}-\ln \left(\frac{b^{2}}{r^{2}}\right)-\frac{7 \kappa c^{2} M}{12 \pi R^{3}} r^{2}\right] \alpha^{2}
$$

We can calculate time component $(B(r))$ of internal metric tensor by integrating the Eq. 13 from specific value of $\mathrm{r}(r<R)$ to surface of $\operatorname{star}(r=R)$. We obtain

$$
B(r)_{i n}=B(R)\left(\frac{\bar{\rho} c^{2}}{P(r)+\bar{\rho} c^{2}}\right)^{2}
$$

where $B(R)$ is the time component of metric at surface and $P(r)$ is pressure at specified radius that can be determined by using numerical integrating the Eq. 16. It is notable that if one insert $r=R$ in Eq. 21 
We can obtain exterior radial and time component of metric, by solving Eqs. 10 and 11 with considering that out of star, $P(r)=\rho(r)=0$. Then for $r>R$, we have

$$
\begin{array}{r}
A(r)_{\text {out }}=B(r)_{\text {out }}=\left[1-\frac{\Lambda r^{2}}{3}-\frac{\kappa c^{2} M}{4 \pi r}\right]+\left[2-\frac{\Lambda r^{2} \ln \left(\frac{b^{2}}{r^{2}}\right)}{3}-\Lambda r^{2}-\ln \left(\frac{b^{2}}{r^{2}}\right)\right. \\
\left.-\frac{7 \kappa c^{2} M}{12 \pi r}+\frac{\kappa c^{2} M}{\pi r} \ln \left(\frac{R}{r}\right)\right] \alpha^{2} .
\end{array}
$$

According to Eqs. 19-22, we can see easily that interior solution matches smoothly to the exterior solution at the surface of the $\operatorname{star}(r=R)$. In Figs. 13 and 14, we have plotted $\frac{1}{A(r)}$ and $B(r)$ against fractional radius $\left(\frac{r}{R}\right.$ ) for different values of $\alpha, \Lambda$ and $B_{b a g}$. We can see that the component of metric at the center of object are non-singular and positive (Shee et al. 2018). Also these figures show that $\frac{1}{A(r)}$ and $B(r)$ are monotonic increasing function of $\frac{r}{R}$ throughout the star as proved by Lake (Lake 2003) for any physically acceptable model.

\section{STABILITY}

\section{1 sound velocity}

When we examine the stability of a compact object in terms of speed of sound, two conditions must be satisfied

(i) The causality condition: Speed of sound $\left(v_{s}\right)$ have to be positive and less than light velocity $(c)$. The variation of $\frac{v_{s}^{2}}{c^{2}}$ with the fractional radius and energy density $\left(\frac{\rho}{\rho_{c}}\right)$ are shown in Figs. 15 and 16. It is observed that sound speed lies between 0 and 1 inside the quark star in all cases. Also $v_{s}$ decreases with increasing (decreasing) the radius (energy density) of star and reach to its minimum value at the surface of star.

(ii) Herrera cracking concept: The cracking concept proposed by Herrera (Herrera 1992) that has been applied to investigating the stability of an anisotropic compact object (Shee et al. 2018; Deb et al. 2017; Maurya et al. 2018). We do not need to check cracking concept, by noting that the model that we have considered here is the isotropic quark star that pressure of star is equal in all directions(see Eq. 9).

\subsection{Adiabatic index}

An essential criterion for the dynamical stability of a compact object against radial adiabatic infinitesimal perturbations, is determined by adiabatic index $\Gamma$

$$
\Gamma=\frac{\rho}{P}\left(1+\frac{P}{\rho c^{2}}\right) \frac{d P}{d \rho} .
$$

Chandrasekhar showed that for the dynamical stability of a compact object, $\Gamma$ must be greater than $\frac{4}{3}$ throughout it (Chandrasekhar 1964). The variation of $\Gamma$ versus radial coordinate $r$ is shown in Figs. 17 and 18 for different cases of $\alpha, \Lambda$ and Bag constant. One can see that the variation of $\alpha$ has no effect on the adiabatic index values while the variation of $\Lambda$ increases the rate of increasing $\Gamma$. In amounts other than the reported value of $\Lambda$, adiabatic index reaches to the greater values near the surface of the star in larger values 
star is closer to and greater than $\frac{4}{3}$ but for layers near the surface are very large. In all cases that we have considered here, the values of $\Gamma$ are higher than $\frac{4}{3}$.

Therefore, we can conclude SQS in dilaton gravity is stable in all values of bag constant that we have considered due to causality condition and adiabatic index inequality $\left(\Gamma \geq \frac{4}{3}\right)$ are valid everywhere inside the star.

\section{CONCLUSION}

We have obtained HEE of a compact object in dilaton gravity by using two approaches and then by using the obtained HEEs we have calculated some structural properties of SQS. We have assumed that the dilaton gravity has constructed from dilaton field with a potential, including two Liouville type terms in the background of Einstein gravity. We have seen that in the values of $\alpha$ and $\Lambda$ where HEE in dilaton gravity has a logical answer, SQS does not change with variations of $\alpha$. This behavior also persists in different values of the bag constant. On the other hand, increasing $\Lambda$ enhances the maximum mass. We have seen that the percentage increase of maximum mass of SQS has higher values for SQSs that are more stable than others. We have shown that effect of dilaton gravity and applying smaller bag constant in EoS of SQM on the SQS has led to existence SQSs with bigger masses and radii that are more stable than SQSs in Einstein gravity. Also, we have shown that SQSs in dilaton gravity are stable against radial adiabatic infinitesimal perturbations in all cases that we have considered here. Moreover, our result show causality condition and energy conditions are valid for the model that has considered. Since the values of $M_{\max }$ and radius for $\Lambda<10^{-14}$ are the same values for $\Lambda=0$ and cosmological observations suggest $\Lambda \leq 3 \times 10^{-56} \mathrm{~cm}^{-2}$, it can be concluded that this limit of the cosmological constant does not affect on SQS structure in dilaton gravity.

Acknowledgements G. H. Bordbar wishes to thank Shiraz University Research Council. This work has been supported by Research Institute for Astronomy and Astrophysics of Maragha.

\section{References}

Alcock, C., Farhi, E., \& Olinto, A. 1986a, Physical Review Letters, 57, 20882

Alcock, C., Farhi, E., \& Olinto, A. 1986b, The Astrophysical Journal, 310, 2612

Alcock, C., \& Olinto, A. 1988, Annual Review of Nuclear and Particle Science, 38, 1612

Arapoğlu, S., Deliduman, C., \& Ekşi, K. Y. 2011, Journal of Cosmology and Astroparticle Physics, 7, 020 2

Astashenok, A. 2016, in International Journal of Modern Physics: Conference Series, Vol. 41, World Scientific, 16601302

Astashenok, A., Capozziello, S., \& Odintsov, S. D. 2015, Physics Letters B, 742, 1602

Astashenok, A. V., Capozziello, S., \& Odintsov, S. D. 2013, Journal of Cosmology and Astroparticle Physics, 12, 0402

Balaguera-Antolínez, A., Nowakowski, M., \& Böhmer, C. G. 2005, International Journal of Modern Physics D, 14, 15074

Böhmer, C. G., \& Harko, T. 2005a, Physics Letters B, 630, 734 
Bombaci, I. 1997, Phys. Rev. C, 55, 15872

Bordbar, G. H., Bigdeli, M., \& Yazdizadeh, T. 2006, International Journal of Modern Physics A, 21, 5991 1

Bordbar, G. H., Hendi, S. H., \& Eslam Panah, B. 2016, The European Physical Journal Plus, 131, 315 1, 6 Bordbar, G., \& Peivand, A. R. 2011, Research in Astronomy and Astrophysics, 11, 851 5, 6

Chan, K., Horne, J., \& Mann, R. 1995, Nuclear Physics B, 447, 441 2, 3

Chandrasekhar, S. 1964, Physical Review Letters, 12, 1149

Cho, Y. 1990, Physical Review D, 41, 24622

Chodos, A., Jaffe, R., Johnson, K., Thorn, C., \& Weisskopf, V. 1974, Physical Review D, 9, 34715

Deb, D., Chowdhury, S., Ray, S., Rahaman, F., \& Guha, B. 2017, Annals of Physics, 387, 239 2, 9

Dehghani, M., \& Farhangkhah, N. 2005, Physical Review D, 71, 0440083

Demorest, P., Pennucci, T., Ransom, S., Roberts, M., \& Hessels, J. 2010, Nature, 467, 10812

Dey, M., Bombaci, I., Dey, J., Ray, S., \& Samanta, B. 1998, Physics Letters B, 438, 1232

Farhi, E., \& Jaffe, R. L. 1984, Physical Review D, 30, 23792

Gangopadhyay, T., Ray, S., Li, X., Dey, J., \& Dey, M. 2013, Monthly Notices of the Royal Astronomical Society, 431, 32162

Glendenning, N. 1990, Modern Physics Letters A, 5, 21972

Güver, T., Wroblewski, P., Camarota, L., \& Özel, F. 2010, The Astrophysical Journal, 719, 18072

Haensel, P., Zdunik, J., \& Schaefer, R. 1986, Astronomy and Astrophysics, 160, 1212

Hendi, S., Bordbar, G., Eslam Panah, B., \& Najafi, M. 2015, Astrophysics and Space Science, 358, 30 2, 3, 5,6

Hendi, S., Bordbar, G., Eslam panah, B., \& Panahiyan, S. 2017, Journal of Cosmology and Astroparticle Physics, 2017, 0042

Hendi, S. H., Faizal, M., Eslam Panah, B., \& Panahiyan, S. 2016, The European Physical Journal C, 76, 2962

Herrera, L. 1992, Physics Letters A, 165, 2069

Katsuragawa, T., Nojiri, S., Odintsov, S., \& Yamazaki, M. 2016, Physical Review D, 93,1240132

Knop et al., R. 2003, The Astrophysical Journal, 598, 1022

Lake, K. 2003, Physical Review D, 67, 1040159

Maurya, S., Ray, S., Ghosh, S., Manna, S., \& T.T., S. 2018, Annals of Physics, 395, 1529

Momeni, D., \& Myrzakulov, R. 2015, International Journal of Geometric Methods in Modern Physics, 12, 15500142

Narain, G., Schaffner-Bielich, J., \& Mishustin, I. N. 2006, Physical Review D, 74, 0630031

Oppenheimer, J. R., \& Volkoff, G. M. 1939, Physical Review, 55, 3741

Paul, B., Chattopadhyay, P., Karmakar, S., \& Tikekar, R. 2011, Modern Physics Letters A, 26, 5752

Perlmutter et al., S. 1999, The Astrophysical Journal, 517, 5652

Rawls, M. L., Orosz, J., McClintock, J., et al. 2011, The Astrophysical Journal, 730, 252

Riess, A., Filippenko, A., Challis, P., et al. 1998, The Astronomical Journal, 116, 10092 
S.H., H., Panah, B. G. B. E., \& Panahiyan, S. 2016, Journal of Cosmology and Astroparticle Physics, 2016, 0132,8

Sharif, M., \& Fatima, H. 2016, International Journal of Modern Physics D, 25, 16500832

Shee, D., Deb, D., Ghosh, S., Ray, S., \& Guha, B. 2018, International Journal of Modern Physics D, 18500899

Sheykhi, A., Riazi, N., \& Mahzoon, M. 2006, Physical Review D, 74, 0440253

Silbar, R. R., \& Reddy, S. 2004, American Journal of Physics, 72, 8921

Stergioulas, N. 2003, Living Reviews in Relativity, 6, 35

Stuchlík, Z. 2000, ACTA PHYSICA SLOVACA, 50, 2194

Tolman, R. C. 1939, Physical Review, 55, 3641

Tonry, J., Schmidt, B., Barris, B., et al. 2003, The Astrophysical Journal, 594, 12

Visser, M., \& Barcelo, C. 2000, in Cosmo-99 (World Scientific), 988

Waga, I. 1993, The Astrophysical Journal, 414, 4366

Witten, E. 1984, Physical Review D, 30, 2722

Zubairi, O., Romero, A., \& Weber, F. 2015, Journal of Physics: Conference Series, 615, 0120036 
Table 1 Maximum gravitational mass $\left(M_{\max }\right)$ and the corresponding radius $(R)$ of SQS in dilaton gravity for various positive values of $\Lambda$ and $\alpha$ with various values of $B_{b a g}$.

\begin{tabular}{ccccccc}
\hline$B_{\text {bag }}\left(\frac{\mathrm{MeV}}{\mathrm{fm}}\right)$ & $\alpha$ & $\Lambda$ & $M_{\max }\left(M_{\odot}\right)$ & $R(\mathrm{Km})$ & $\bar{\rho}\left(10^{15} \frac{\mathrm{gr}}{\mathrm{cm}}\right)$ & $z\left(10^{-1}\right)$ \\
\hline & 0 & 0 & 1.55 & 8.86 & 1.06 & 4.37 \\
60 & $1.00 \times 10^{-8}$ & 0 & 1.55 & 8.86 & 1.06 & 4.37 \\
& $1.00 \times 10^{-5}$ & 0 & 1.55 & 8.86 & 1.06 & 4.37 \\
& 0 & $1.00 \times 10^{-56}$ & 1.55 & 8.86 & 1.06 & 4.37 \\
& $1.00 \times 10^{-8}$ & $1.00 \times 10^{-56}$ & 1.55 & 8.86 & 1.06 & 4.37 \\
& $1.00 \times 10^{-5}$ & $1.00 \times 10^{-56}$ & 1.55 & 8.86 & 1.06 & 4.37 \\
\hline \multirow{4}{*}{75} & 0 & 0 & 1.43 & 8.15 & 1.25 & 4.40 \\
& $1.00 \times 10^{-8}$ & 0 & 1.43 & 8.15 & 1.25 & 4.40 \\
& $1.00 \times 10^{-5}$ & 0 & 1.43 & 8.15 & 1.25 & 4.40 \\
& 0 & $1.00 \times 10^{-56}$ & 1.43 & 8.15 & 1.25 & 4.40 \\
& $1.00 \times 10^{-8}$ & $1.00 \times 10^{-56}$ & 1.43 & 8.15 & 1.25 & 4.40 \\
& $1.00 \times 10^{-5}$ & $1.00 \times 10^{-56}$ & 1.43 & 8.15 & 1.25 & 4.40 \\
\hline \multirow{4}{*}{90} & 0 & 0 & 1.34 & 7.58 & 1.46 & 4.45 \\
& $1.00 \times 10^{-8}$ & 0 & 1.34 & 7.58 & 1.46 & 4.45 \\
& $1.00 \times 10^{-5}$ & 0 & 1.34 & 7.58 & 1.46 & 4.45 \\
& 0 & $1.00 \times 10^{-56}$ & 1.34 & 7.58 & 1.46 & 4.45 \\
& $1.00 \times 10^{-8}$ & $1.00 \times 10^{-56}$ & 1.34 & 7.58 & 1.46 & 4.45 \\
& & $1.00 \times 10^{-56}$ & 1.34 & 7.58 & 1.46 & 4.45 \\
\hline
\end{tabular}

Table 2 Maximum gravitational mass $\left(M_{\max }\right)$ and the corresponding radius $(R)$ of SQS in dilaton gravity for various positive values of $\Lambda$ at $b=10^{-4}$ and $\alpha=0$

\begin{tabular}{lccccccc}
\hline$\Lambda$ & $M_{\max }\left(M_{\odot}\right)$ & $R(K m)$ & $M_{\max }^{\text {eff }}\left(M_{\odot}\right)$ & $R^{\text {eff }}(\mathrm{Km})$ & $\left.\bar{\rho}\left(10^{15} \mathrm{gr} \mathrm{cm}\right)^{-3}\right)$ & $z_{\text {eff }}\left(10^{-1}\right)$ & $z\left(10^{-1}\right)$ \\
\hline 0 & 1.34 & 7.58 & 1.34 & 7.58 & 1.46 & 4.45 & 4.45 \\
$1.00 \times 10^{-14}$ & 1.34 & 7.58 & 1.34 & 7.58 & 1.43 & 4.48 & 4.48 \\
$1.00 \times 10^{-13}$ & 1.36 & 7.68 & 1.36 & 7.68 & 1.43 & 4.78 & 4.78 \\
$3.00 \times 10^{-13}$ & 1.43 & 7.95 & 1.43 & 7.95 & 1.35 & 5.69 & 5.69 \\
$4.00 \times 10^{-13}$ & 1.47 & 8.13 & 1.47 & 8.13 & 1.30 & 6.25 & 6.25 \\
$5.00 \times 10^{-13}$ & 1.52 & 8.34 & 1.52 & 8.34 & 1.24 & 6.99 & 6.99 \\
$8.00 \times 10^{-13}$ & 1.86 & 10.01 & 1.86 & 10.01 & 0.88 & 13.27 & 13.27 \\
\hline
\end{tabular}

Table 3 Maximum gravitational mass $\left(M_{\max }\right)$ and the corresponding radius $(R)$ of SQS in dilaton gravity for various positive values of $\Lambda$ and $\alpha$ with $B_{b a g}=60 \frac{\mathrm{MeV}}{\mathrm{fm}^{3}}$.

\begin{tabular}{lcccccc}
\hline$\alpha$ & $\Lambda$ & $M_{\max }\left(M_{\odot}\right)$ & $R(K m)$ & $\bar{\rho}\left(10^{15} \frac{\mathrm{gr}}{\mathrm{cm}}\right)$ & $z\left(10^{-1}\right)$ & $B_{\text {bag }}\left(\frac{\mathrm{MeV}}{\mathrm{fm}}\right)$ \\
\hline $1.00 \times 10^{-7}$ & $1.00 \times 10^{-15}$ & 1.55 & 8.86 & 1.06 & 4.38 & 60 \\
$1.00 \times 10^{-7}$ & $1.00 \times 10^{-14}$ & 1.55 & 8.88 & 1.06 & 4.41 & 60 \\
0 & $1.00 \times 10^{-13}$ & 1.59 & 9.04 & 1.02 & 4.84 & 60 \\
0 & $3.00 \times 10^{-13}$ & 1.71 & 9.53 & 0.94 & 6.22 & 60 \\
0 & $4.00 \times 10^{-13}$ & 1.78 & 9.87 & 0.88 & 7.20 & 60 \\
0 & $5.00 \times 10^{-13}$ & 1.90 & 10.43 & 0.80 & 8.85 & 60 \\
$1.00 \times 10^{-8}$ & $5.00 \times 10^{-13}$ & 1.90 & 10.43 & 0.80 & 8.85 & 60 \\
$1.00 \times 10^{-6}$ & $5.00 \times 10^{-13}$ & 1.90 & 10.43 & 0.80 & 8.85 & 60 \\
\hline
\end{tabular}


Table 4 Maximum gravitational mass $\left(M_{\max }\right)$ and the corresponding $\operatorname{radius}(R)$ of SQS in dilaton gravity for various positive values of $\Lambda$ and $\alpha$ with $B_{b a g}=75 \frac{\mathrm{MeV}}{\mathrm{fm}^{3}}$.

\begin{tabular}{lcccccc}
\hline$\alpha$ & $\Lambda$ & $M_{\max }\left(M_{\odot}\right)$ & $R(K m)$ & $\bar{\rho}\left(10^{15} \frac{\mathrm{gr}}{\mathrm{cm}^{3}}\right)$ & $z\left(10^{-1}\right)$ & $B_{\text {bag }}\left(\frac{\mathrm{MeV}}{\mathrm{fm}}\right)$ \\
\hline $1.00 \times 10^{-7}$ & $1.00 \times 10^{-15}$ & 1.43 & 8.15 & 1.25 & 4.40 & 75 \\
$1.00 \times 10^{-7}$ & $1.00 \times 10^{-14}$ & 1.44 & 8.15 & 1.26 & 4.48 & 75 \\
$1.00 \times 10^{-8}$ & $1.00 \times 10^{-13}$ & 1.46 & 8.30 & 1.21 & 4.77 & 75 \\
0 & $1.00 \times 10^{-13}$ & 1.46 & 8.30 & 1.21 & 4.77 & 75 \\
0 & $3.00 \times 10^{-13}$ & 1.55 & 8.63 & 1.15 & 5.90 & 75 \\
0 & $4.00 \times 10^{-13}$ & 1.60 & 8.87 & 1.09 & 6.60 & 75 \\
0 & $5.00 \times 10^{-13}$ & 1.67 & 9.18 & 1.03 & 7.60 & 75 \\
$1.00 \times 10^{-8}$ & $5.00 \times 10^{-13}$ & 1.67 & 9.18 & 1.03 & 7.60 & 75 \\
$1.00 \times 10^{-6}$ & $5.00 \times 10^{-13}$ & 1.67 & 9.18 & 1.03 & 7.60 & 75 \\
\hline
\end{tabular}

Table 5 The percentage increase of maximum mass of SQS in dilaton gravity for various values of $\Lambda$ at $b=10^{-4}$ and $\alpha=0$ with different values of $B_{b a g}$.

\begin{tabular}{lcccr}
\hline $\mathbf{B}_{\text {bag }}$ & $(\boldsymbol{\Delta} \mathbf{M})_{\Lambda_{1}}\left[M_{\max \Lambda_{1}}\right]$ & $(\Delta \mathbf{M})_{\Lambda_{\mathbf{2}}}\left[M_{\max \Lambda_{2}}\right]$ & $(\Delta \mathbf{M})_{\Lambda_{3}}\left[M_{\max \Lambda_{3}}\right]$ & $(\Delta \mathbf{M})_{\Lambda_{4}}\left[M_{\max \Lambda_{4}}\right]$ \\
& $\left(\Lambda_{1}=1.00 \times 10^{-13}\right)$ & $\left(\Lambda_{2}=3.00 \times 10^{-13}\right)$ & $\left(\Lambda_{3}=4.00 \times 10^{-13}\right)$ & $\left(\Lambda_{4}=5.00 \times 10^{-13}\right)$ \\
\hline 90 & $1.49 \%$ & $6.72 \%$ & $9.70 \%$ & $13.43 \%$ \\
75 & $2.10 \%$ & $8.39 \%$ & $11.89 \%$ & $16.78 \%$ \\
60 & $2.58 \%$ & $10.32 \%$ & $14.84 \%$ & $22.58 \%$ \\
\hline
\end{tabular}

Table 6 Maximum gravitational mass $\left(M_{\max }\right)$ and the corresponding radius $(R)$ of SQS in dilaton gravity for various negative values of $\Lambda$ at $b=10^{-4}$ and $\alpha=0$.

\begin{tabular}{lccr}
\hline$\alpha$ & $\Lambda$ & $M_{\max }\left(M_{\odot}\right)$ & $R(K m)$ \\
\hline 0 & 0 & 1.34 & 7.57 \\
0 & $-8.00 \times 10^{-13}$ & 1.19 & 6.94 \\
0 & $-1.00 \times 10^{-12}$ & 1.16 & 6.83 \\
\hline
\end{tabular}




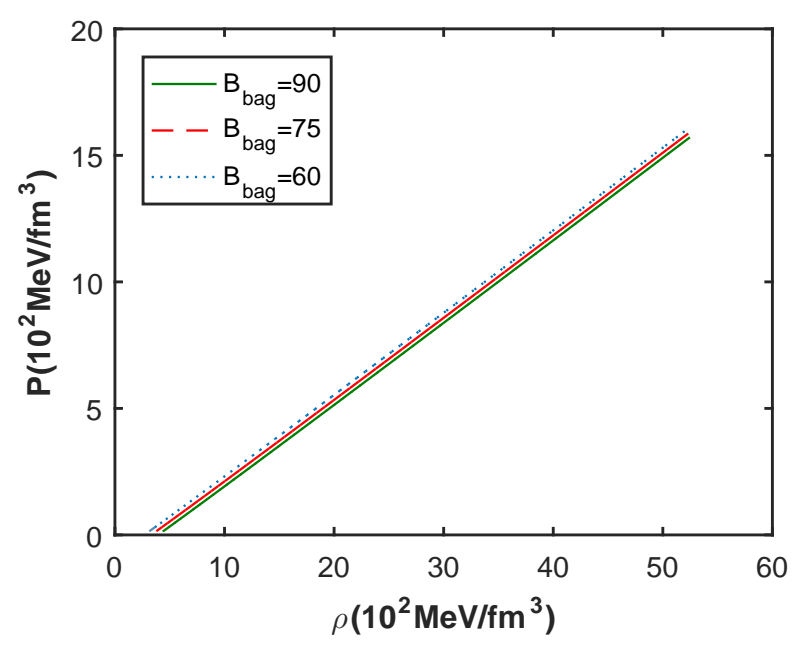

a)

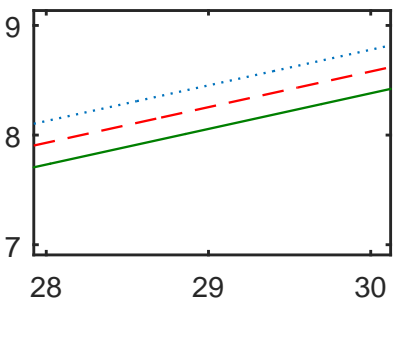

b)

Fig. 1 EoS of SQM for various values of Bag constant(left panel). The right panel shows a zoom of the left one.

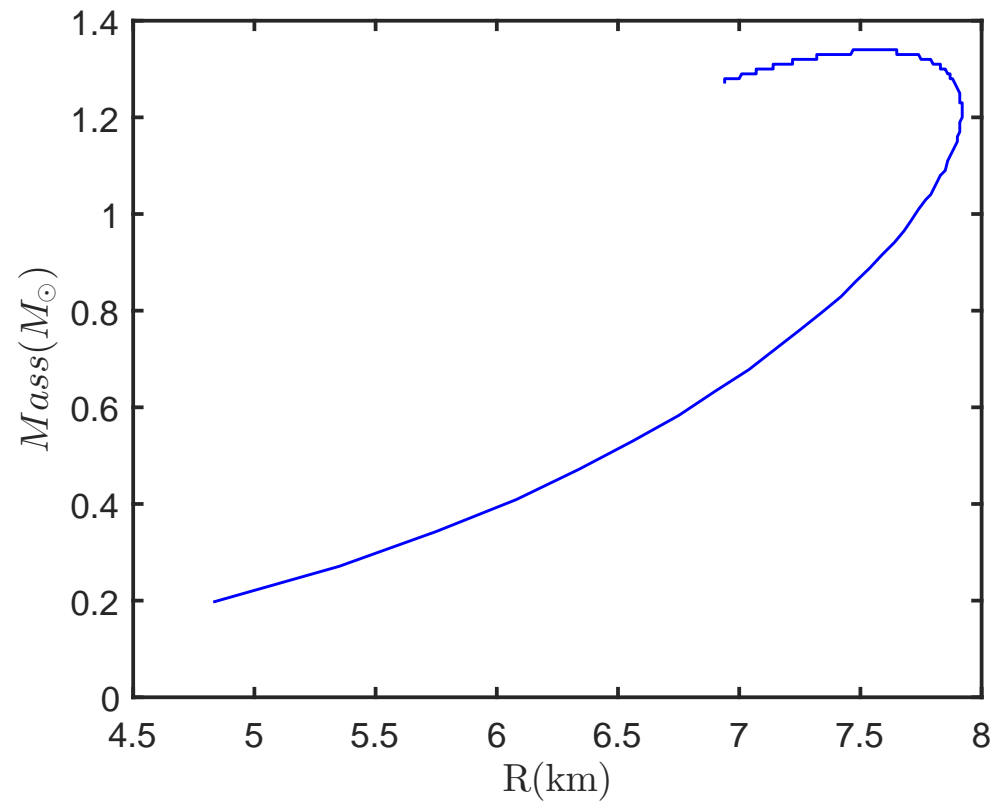

Fig. 2 The mass-radius relation of SQS for $\Lambda=0\left(\alpha=0, b=10^{-4}\right.$ and $\left.B_{b a g}=90 \frac{\mathrm{MeV}}{\mathrm{fm}^{3}}\right)$. 


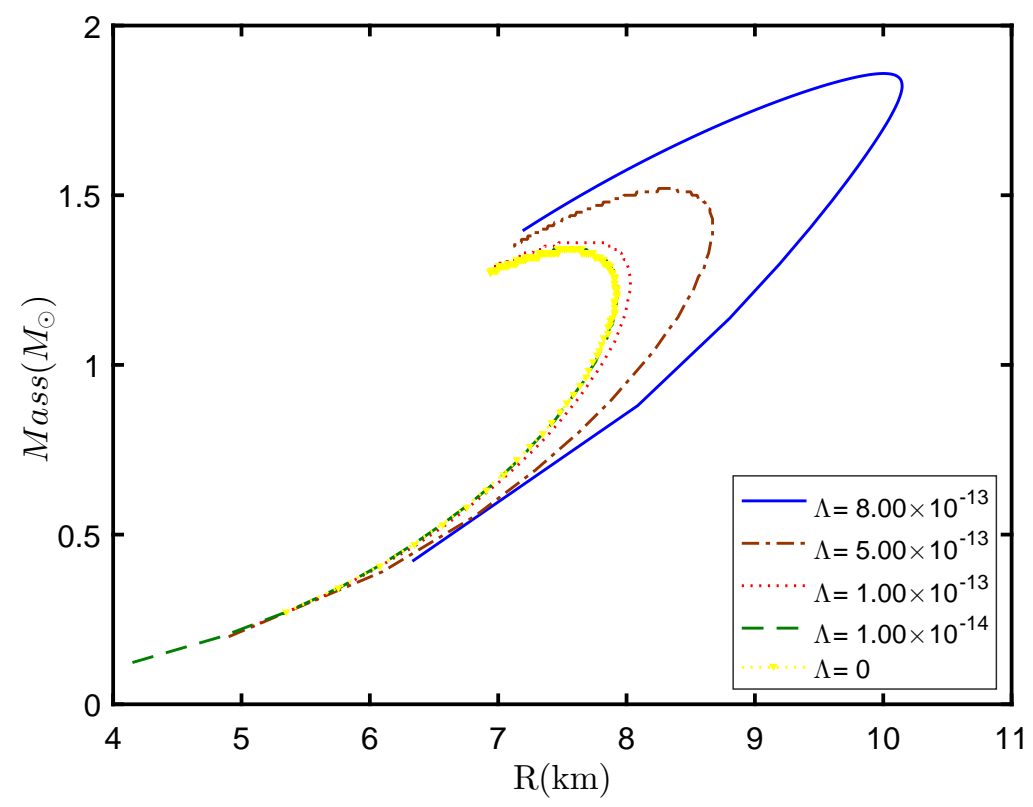

Fig. 3 The mass-radius relation of SQS for various positive values of $\Lambda$ ( $\alpha=0, b=$ $\left.10^{-4}, B_{b a g}=90 \frac{\mathrm{MeV}}{\mathrm{fm}^{3}}\right)$

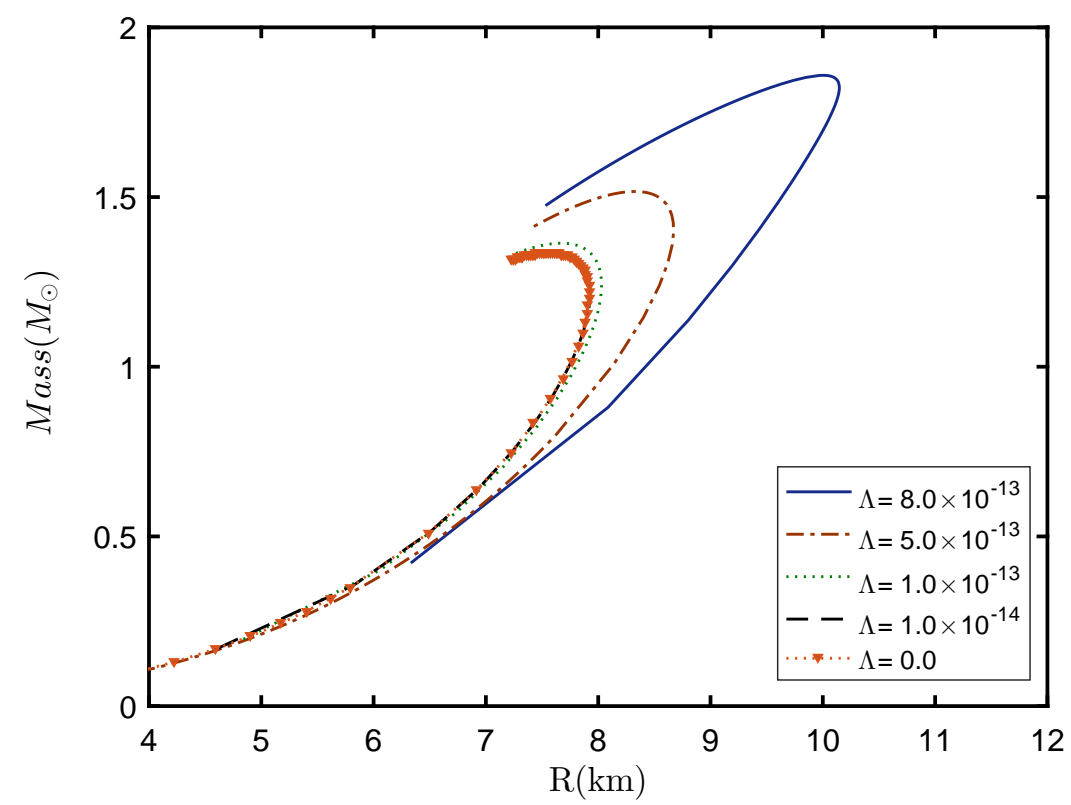

Fig. 4 The effective mass-effective radius relation of SQS for various positive values of $\Lambda$ ( $\alpha=0$, $\left.b=10^{-4}, B_{\text {bag }}=90 \frac{\mathrm{MeV}}{\mathrm{fm}^{3}}\right)$ 


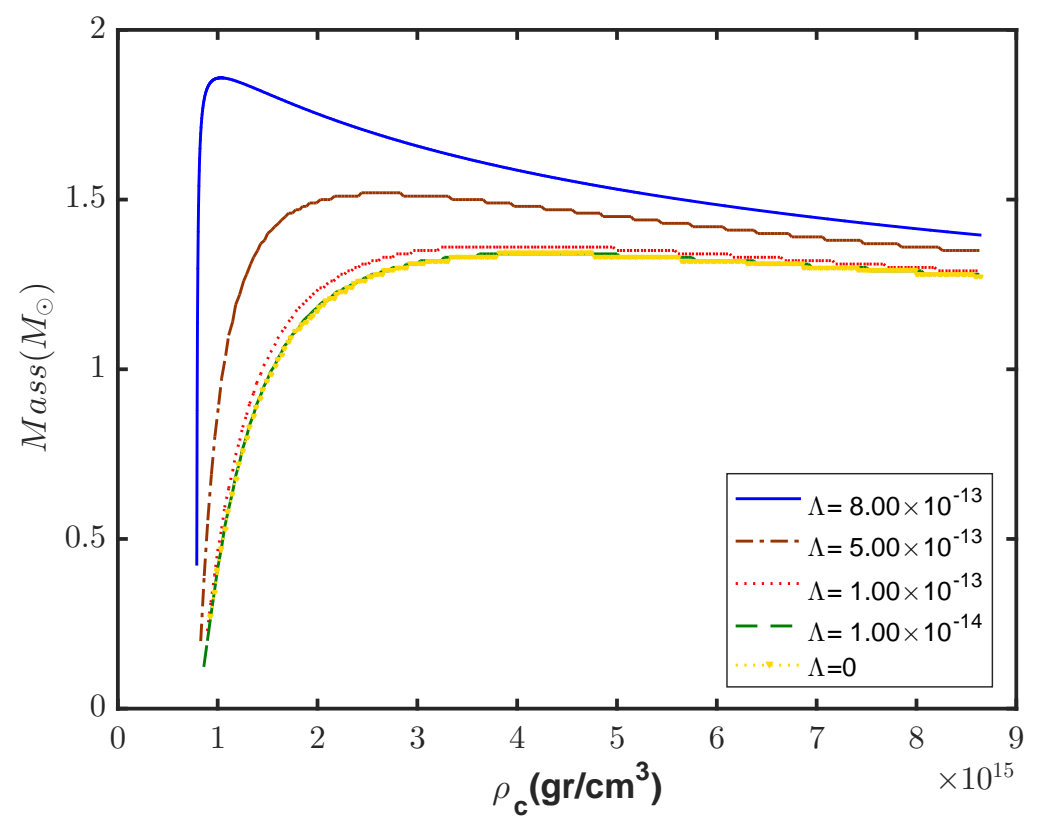

Fig. 5 The gravitational mass versus the total central energy density of the SQS with various values of $\Lambda$ at $\alpha=0, b=10^{-4}$ and $B_{b a g}=90 \frac{\mathrm{Mev}}{\mathrm{fm}^{3}}$

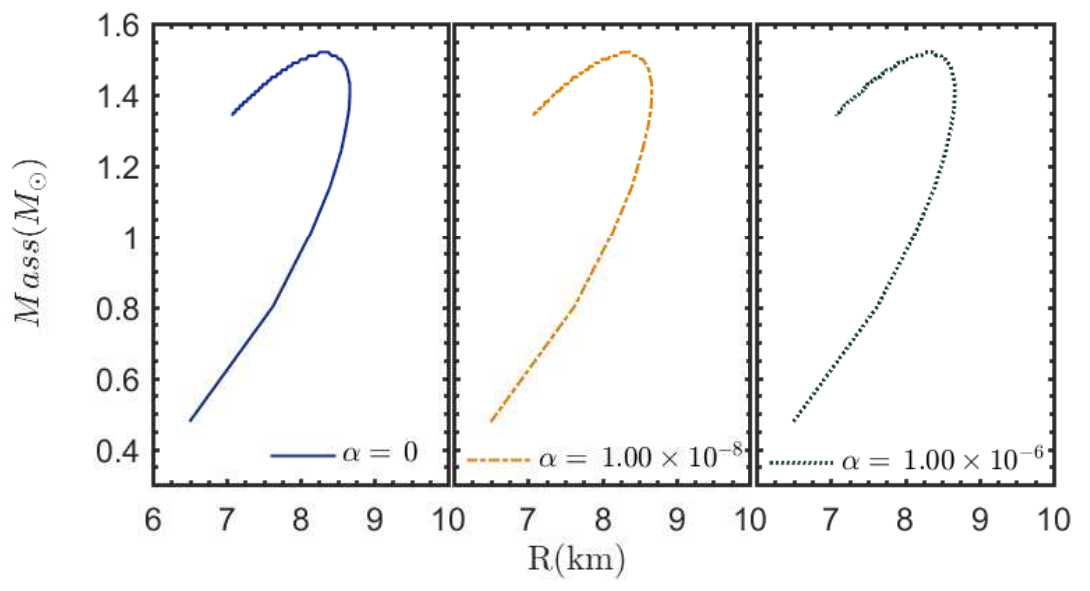

(a)

(b)

(c)

Fig. 6 The mass-radius relation of SQS for $\Lambda=5.00 \times 10^{-13}$ at (a) $\alpha=0$ (b) $\alpha=1.00 \times 10^{-8}$ (c) $\alpha=1.00 \times 10^{-6}$ 


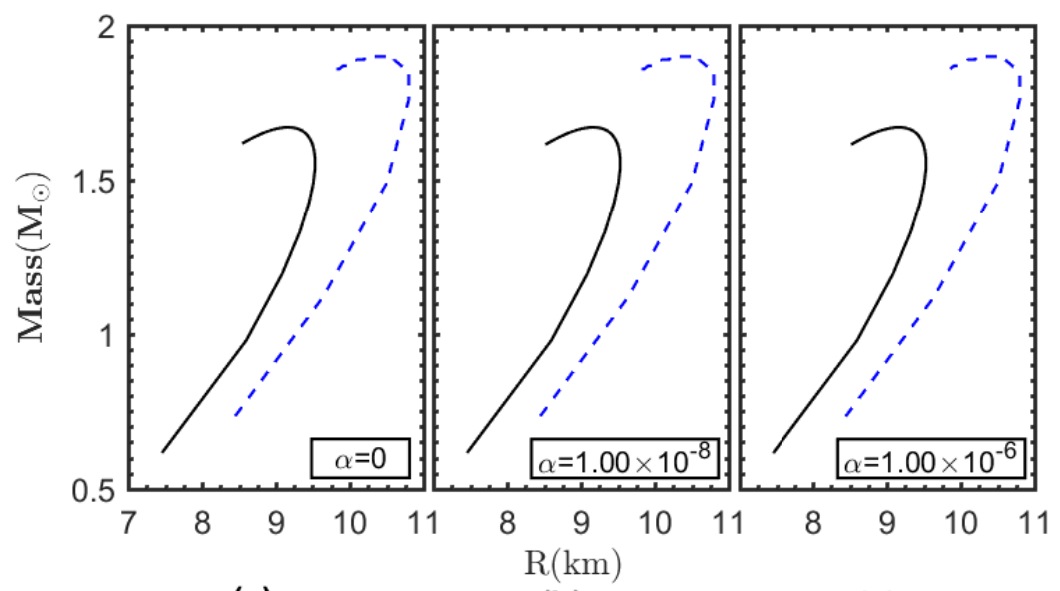

(a)

(b)

(c)

Fig. 7 As Fig. 6,but for $B_{b a g}=60 \frac{\mathrm{MeV}}{\mathrm{fm}^{3}}$ (dashed lines) and $B_{b a g}=75 \frac{\mathrm{MeV}}{\mathrm{fm}^{3}}$ (solid lines) .

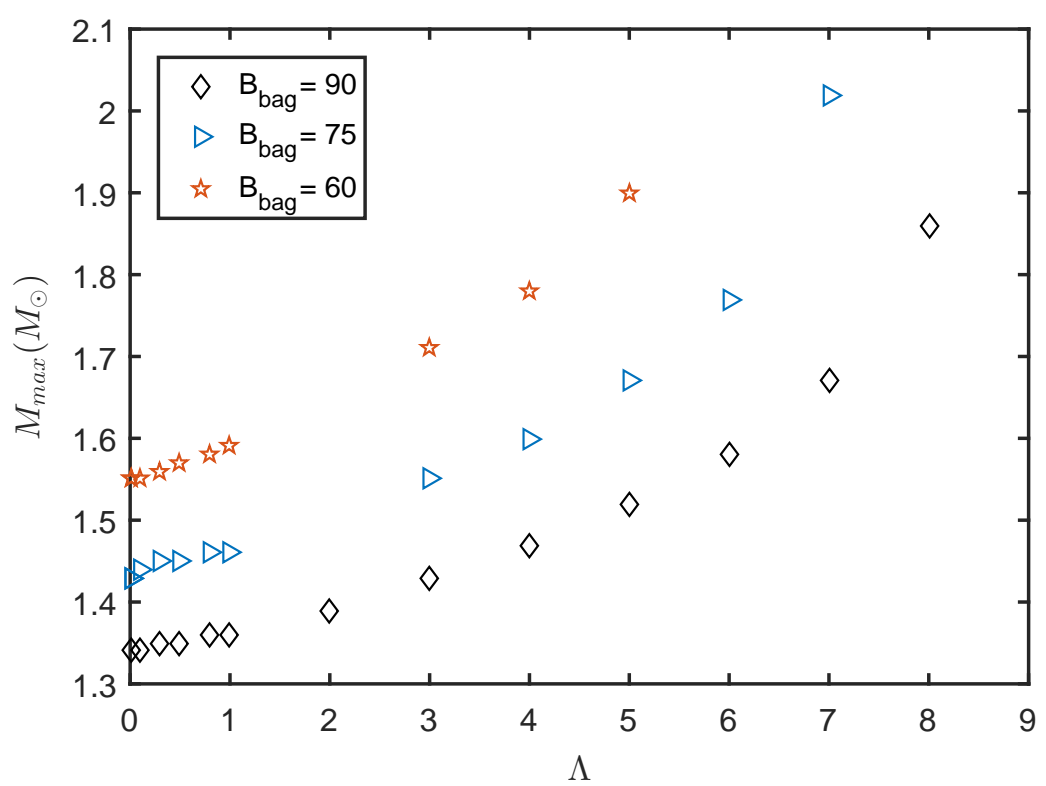

Fig. 8 Maximum mass of SQS versus cosmological constant $\left(\times 10^{-13}\right)$ for different bag constant with $\alpha=0$. 


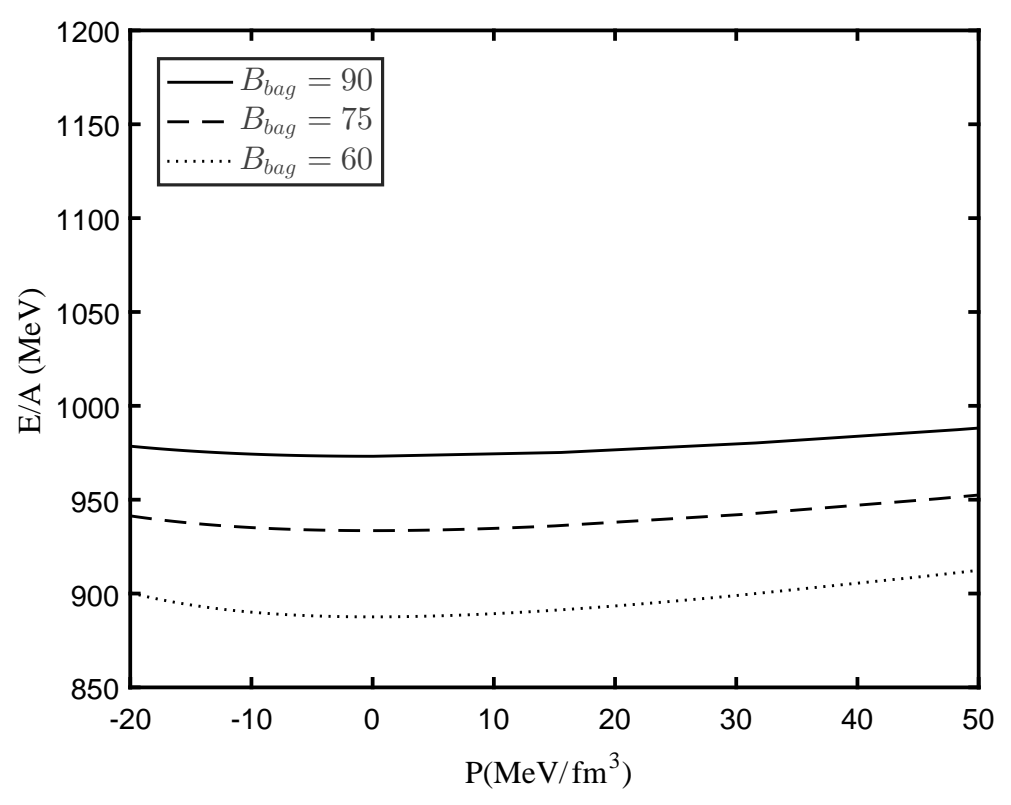

Fig. 9 The energy per baryon versus the pressure $(P)$ for SQSs with the various values of bag constant.

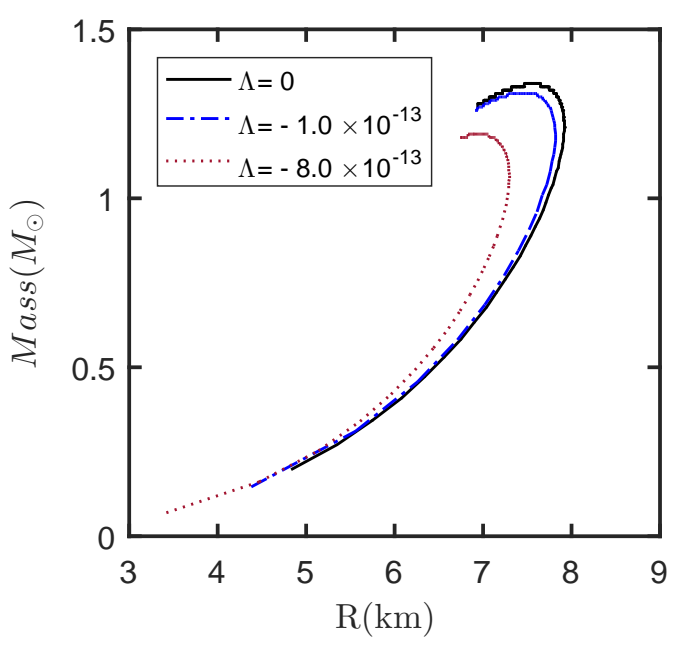

(a)

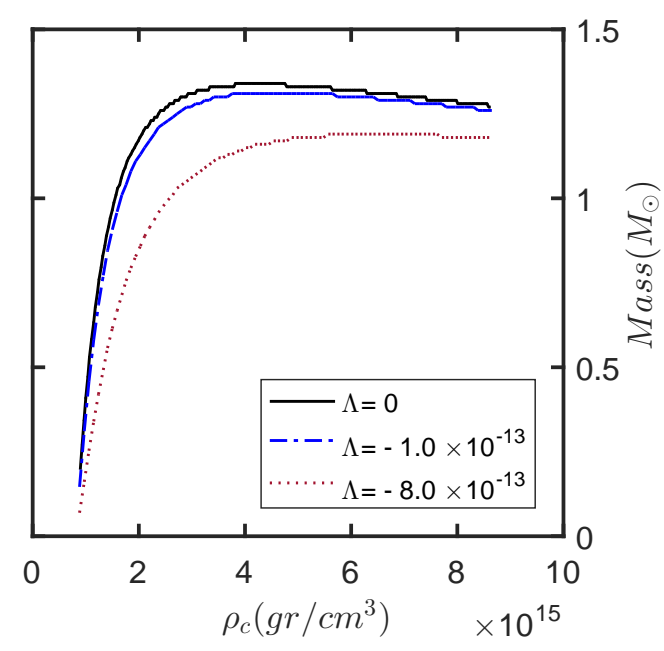

(b)

Fig. 10 The gravitational mass versus a)corresponding radius and b)central energy density $\left(\rho_{c}\right)$ of SQS in dilaton gravity for negative values of $\Lambda\left(\alpha=0, b=10^{-4}\right.$ and $\left.B_{b a g}=90 \frac{\mathrm{MeV}}{\mathrm{fm}^{3}}\right)$. 

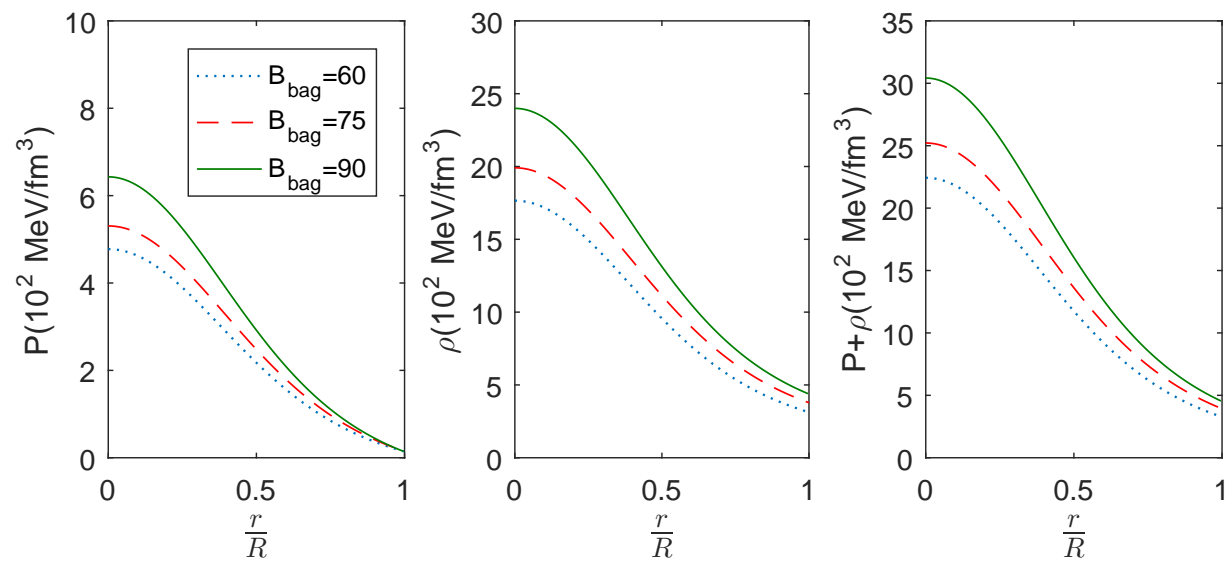

Fig. 11 The behavior of pressure $P$, energy density $\rho$ and $P+\rho$ with respect to fractional radius $\frac{r}{R}$ under various values of $B_{b a g}$ with $\alpha=\Lambda=0$.
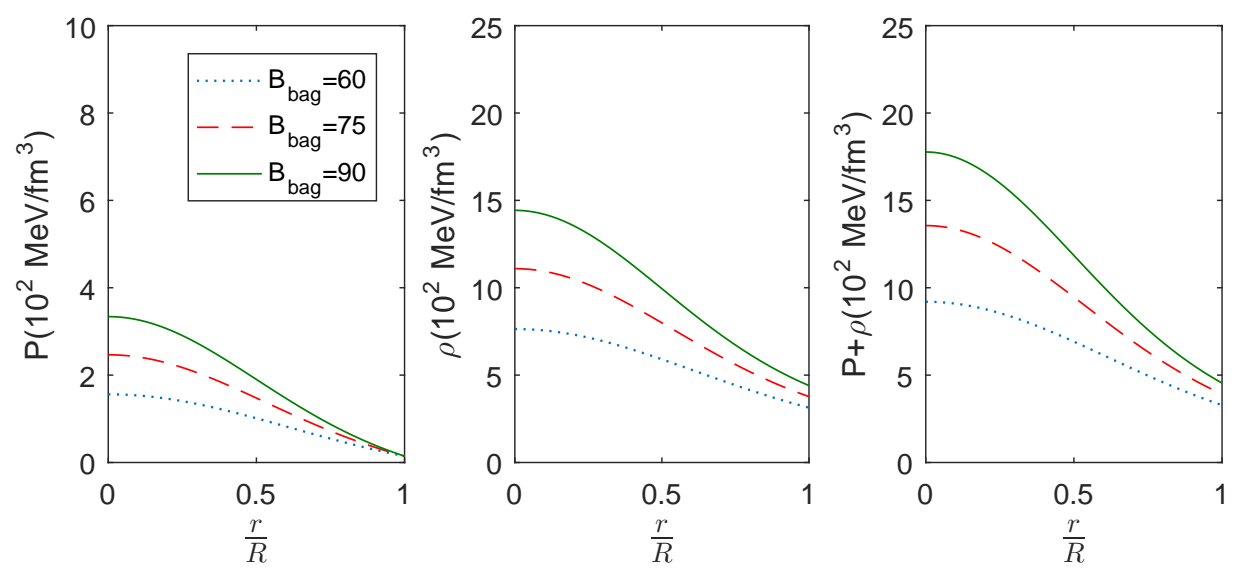

Fig. 12 As Fig. 11 for $\alpha=1 \times 10^{-6}$ and $\Lambda=5 \times 10^{-13}$. 

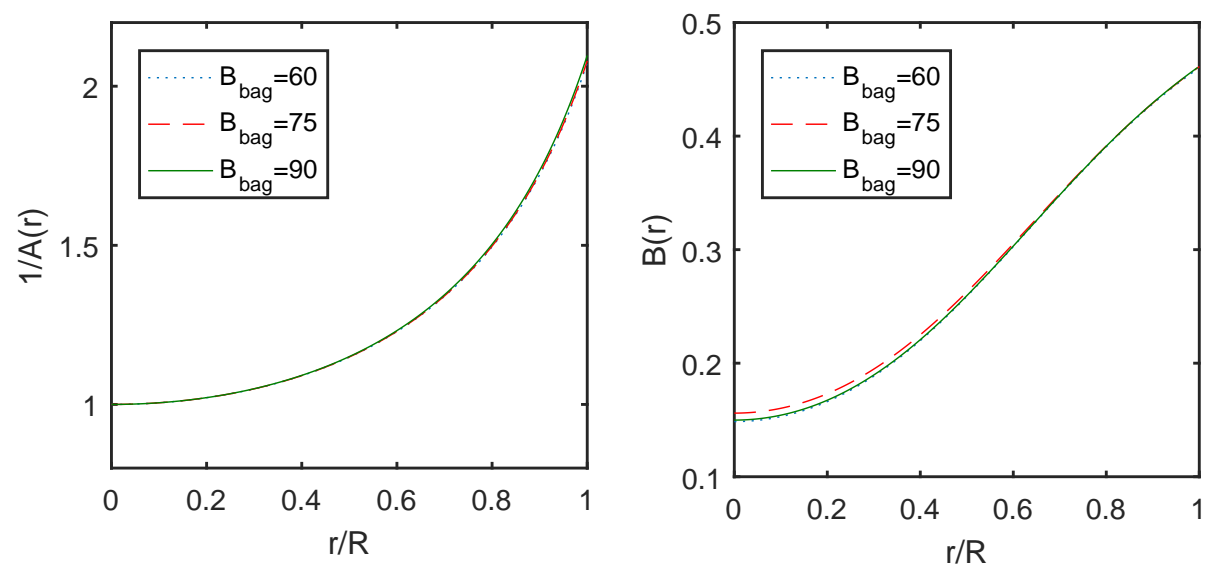

Fig. 13 The variation in radial and time component of metric tensor with respect to fractional radius $\frac{r}{R}$ under various values of $B_{b a g}$ with $\alpha=\Lambda=0$.
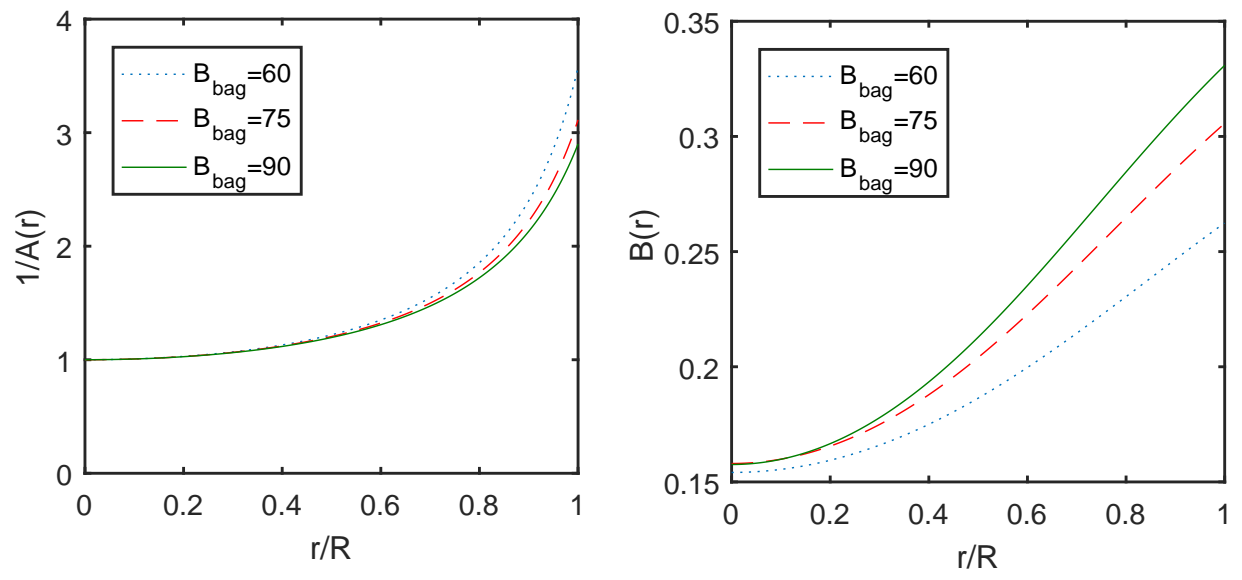

Fig. 14 As Fig. 13 but for $\alpha=1 \times 10^{-6}$ and $\Lambda=5 \times 10^{-13}$. 

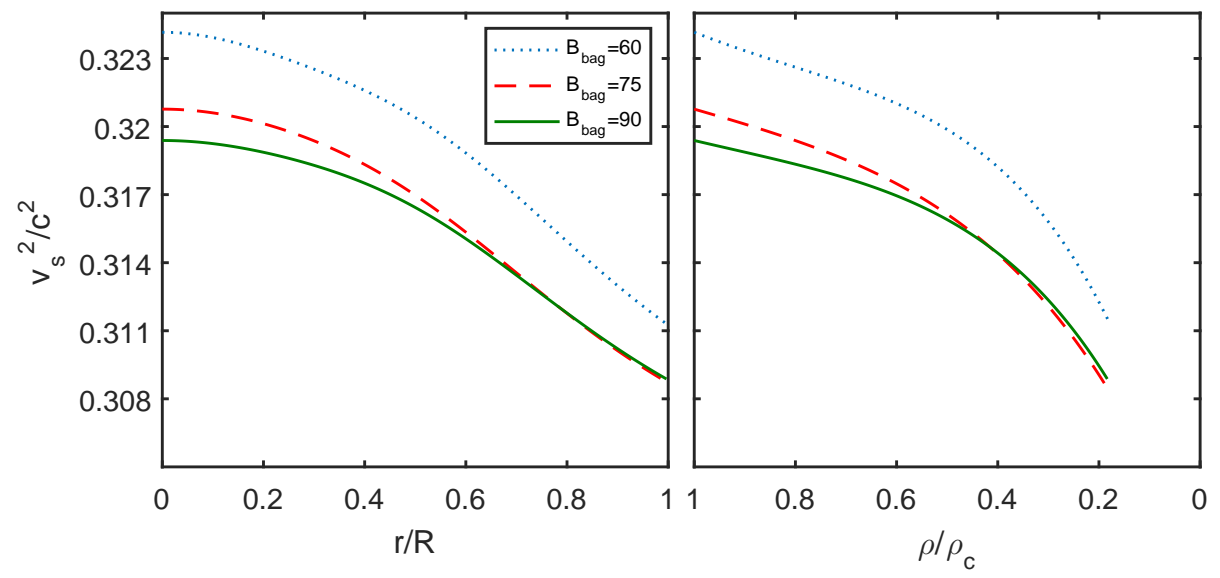

Fig. 15 The behavior of $\frac{v_{s}^{2}}{c^{2}}$ with respect to fractional radius $\frac{r}{R}$ (left panel) and fractional energy density $\frac{\rho}{\rho_{c}}$ (right panel) under various values of $B_{b a g}$ with $\alpha=\Lambda=0$.
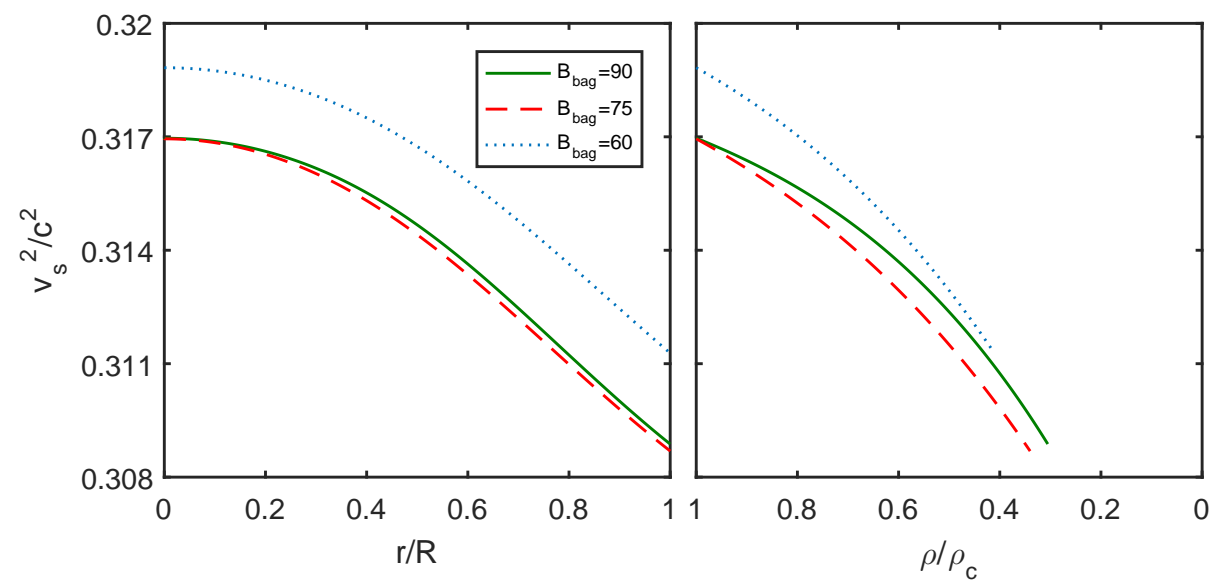

Fig. 16 As Fig. 15 but for $\alpha=1 \times 10^{-6}$ and $\Lambda=5 \times 10^{-13}$. 

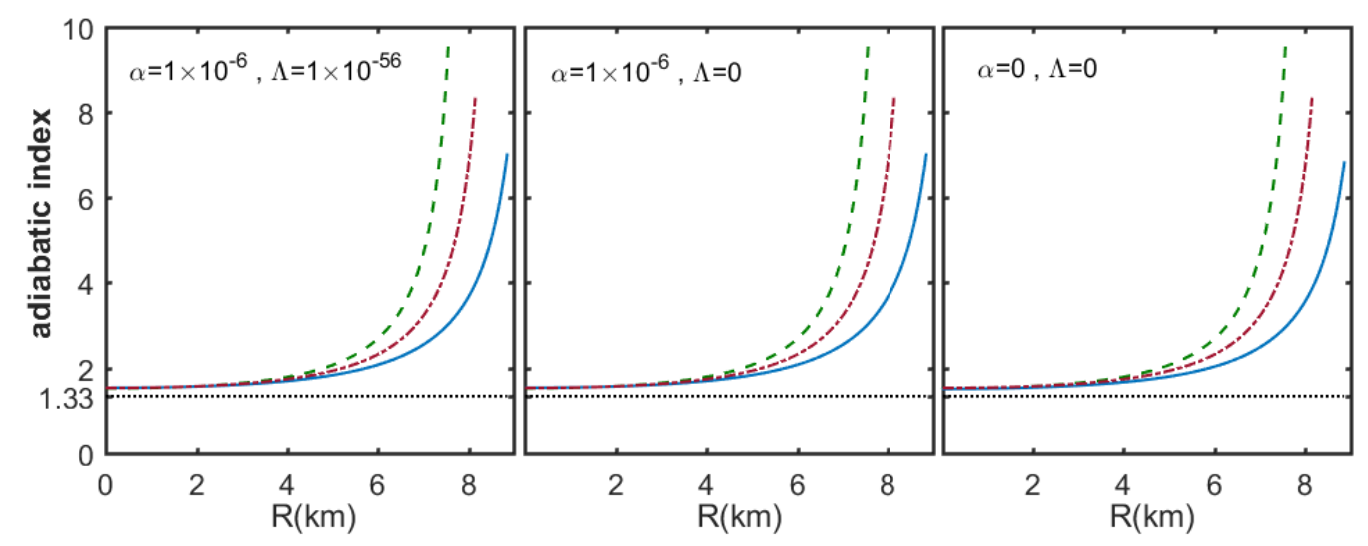

Fig. 17 The variation in adiabatic index with radial coordinate $r$ for different cases of $\alpha$ and $\Lambda$ with $B_{b a g}=60 \frac{\mathrm{MeV}}{\mathrm{fm}^{3}}$ (solid line), $B_{b a g}=75 \frac{\mathrm{MeV}}{\mathrm{fm}^{3}}$ (Dash-dot line) and $B_{b a g}=90 \frac{\mathrm{MeV}}{\mathrm{fm}^{3}}$ (dashed line).

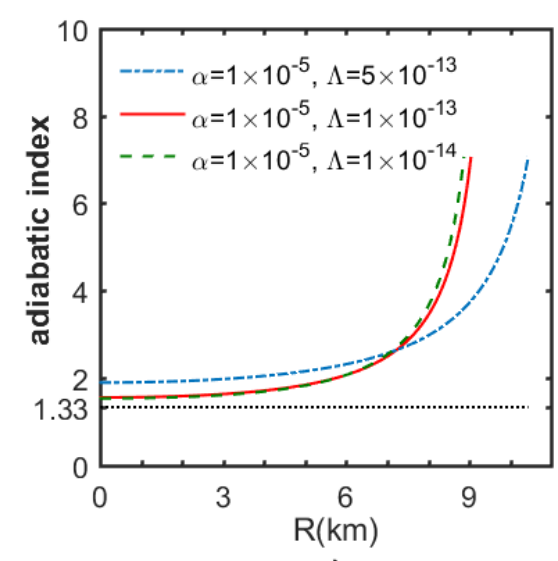

a)

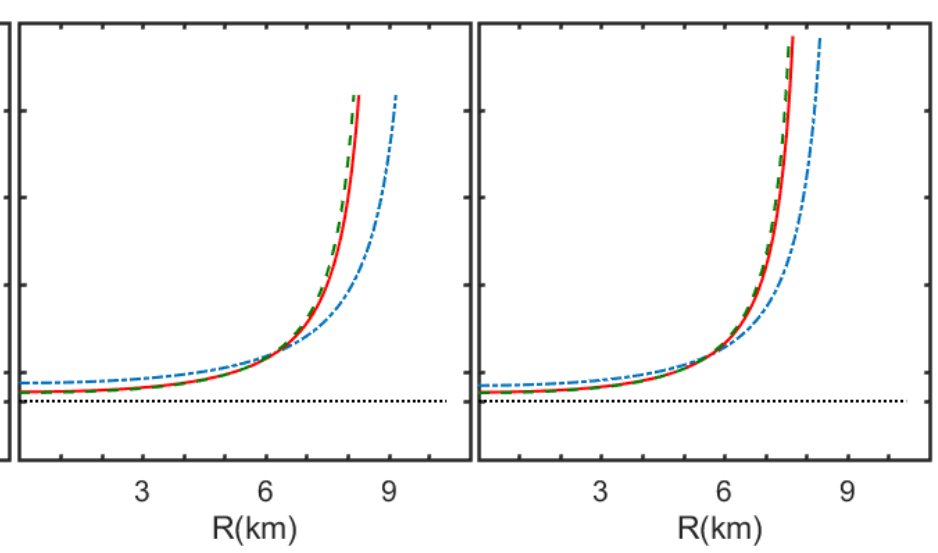

b)

c)

Fig. 18 The variation in adiabatic index with radial coordinate $\mathrm{r}$ under various condition of $\alpha$ and $\Lambda$ in(a) $B_{b a g}=60 \frac{\mathrm{MeV}}{\mathrm{fm}^{3}}$, (b) $B_{b a g}=75 \frac{\mathrm{MeV}}{\mathrm{fm}^{3}}$ and (c) $B_{b a g}=90 \frac{\mathrm{MeV}}{\mathrm{fm}^{3}}$ 